\title{
Modifizierte Verschiebelappenplastik nach von Burow für zwei runde und verbundene Hautdefekte - die 8-zu-S-Plastik
}

Modified Burow's-Advancement-Flaps for Two Circular and Confluent Cutanous Defects - The 8-to-S-Plasty

\section{Zusammenfassung}

Die spindelförmige Exzision mit Dehnungsplastik oder die Verschiebelappenplastik nach von Burow sind die üblichen operativen Defektdeckungen zweier runder und verbundener oder aneinanderliegender Defekte (8-förmige Defekte). Bei beiden plastischen Hautoperationen wird jedoch gesunde Haut geopfert (Ausgleichsdreiecke). Wir berichten über eine modifizierte Verschiebelappenplastik nach von Burow (8-zu-S-Plastik), die ohne zusätzliche Inzisionen auskommt. Die modifizierte Operationstechnik führt zu kürzeren Narben verglichen zur traditionellen Burow-Verschiebelappenplastik.

Die Nahtverschlusslinie erinnert an ein S.

\section{Abstract}

The traditional way to close two confluent or closely approximated cutaneous defects (namely a 8-shaped-defect) sacrifices healthy skin and does not give optimal aesthetic results. We report a case with modified Burow's-advancementflaps describing less sacrificed skin and shorter scars. Modified Burow's-advancement-flaps (8-to-S-Plasty) were applied sparing two Burow skin triangles. Application of that modified operation technique leads to shorter scars if compared to traditional Burow's-advancement-flaps. A modified technique is presented here for skin defects shaped like an 8 - namely two similar round and confluent lesions. The suture line after closing resembles an S. With this 8-to-S-Plasty no additional incisions are necessary.

\section{Einleitung}

Die Verschiebelappenplastik nach von Burow oder die spindelförmige Exzision sind bisherige Verfahren zum operativen Verschluss von 8-förmigen Defekten (Abb.1).

Aber in beiden Fällen muss gesunde Haut geopfert werden und müssen zusätzliche Inzisionen erfolgen, um die Ausgleichsdreiecke zu exzidieren. Um gesunde Haut zu schonen und einen 8-förmigen Defekt zu rekonstruieren, entwickelten wir eine modifizierte Operationstechnik. Diese besteht aus einer modifizierten Verschiebelappenplastik nach von Burow. In unserem Fall führte die neue Technik zu einem guten ästhetischen Ergebnis und zu kürzeren Narben.

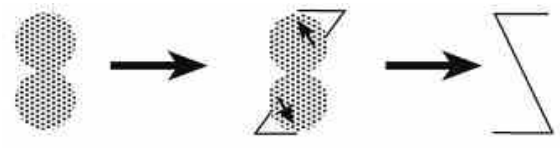

a

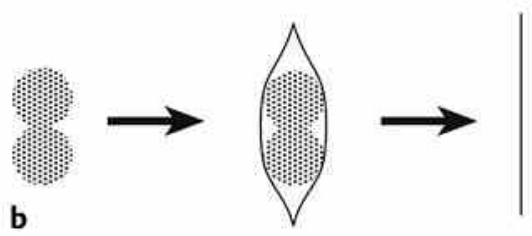

Abb. 1 Traditionelle Burow-Verschiebelappenplastik (a) und elliptische Exzision (b) zum Verschluss eines 8-förmigen Defekts. Links Inzisionslinien; rechts Nahtlinien. 


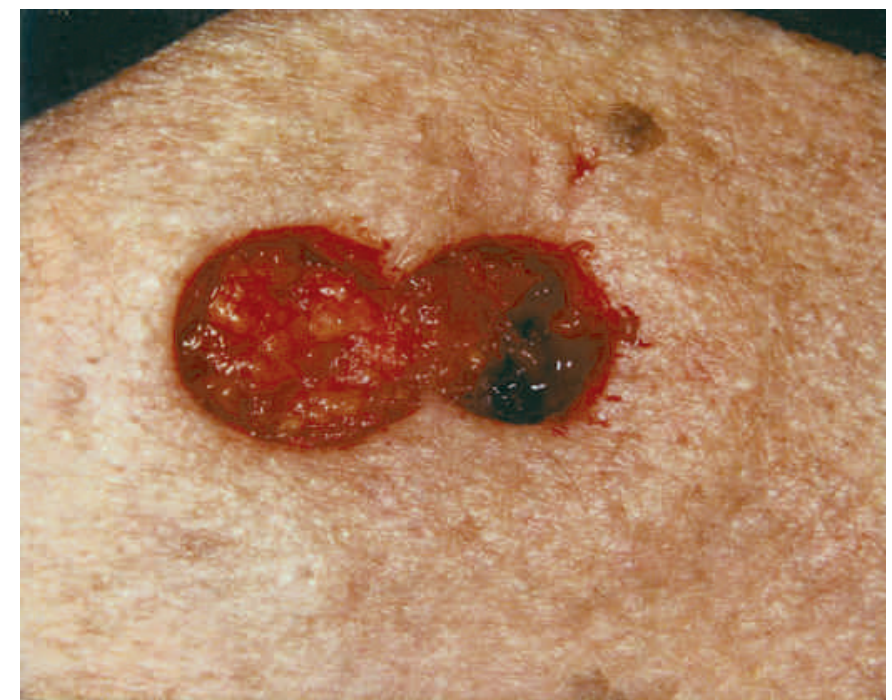

Abb. 2 Der 8-förmige Defekt (Durchmesser 25 mm und $23 \mathrm{~mm}$ )
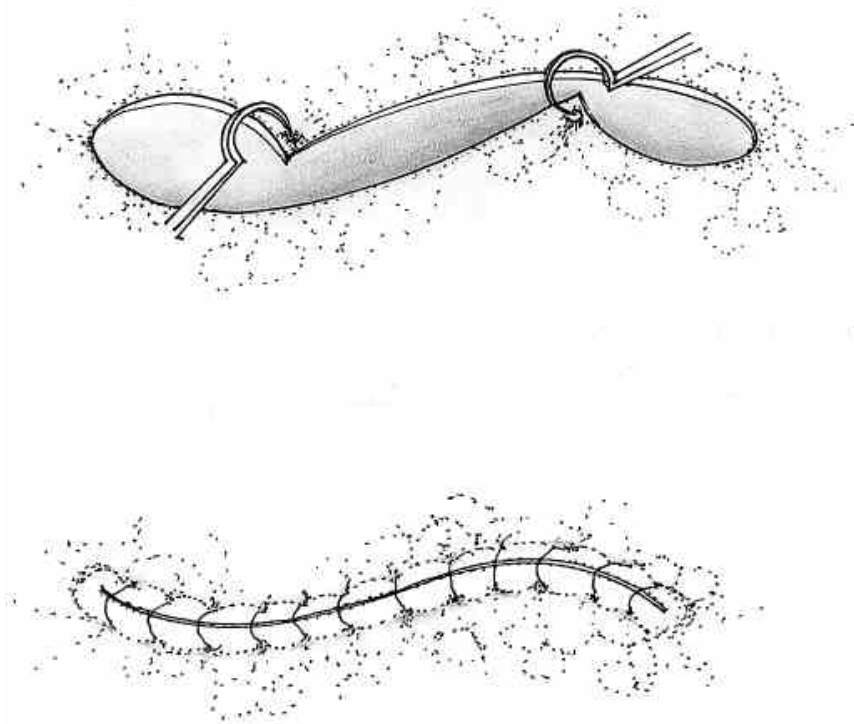

Abb. 3 Die 8-zu-S-Plastik

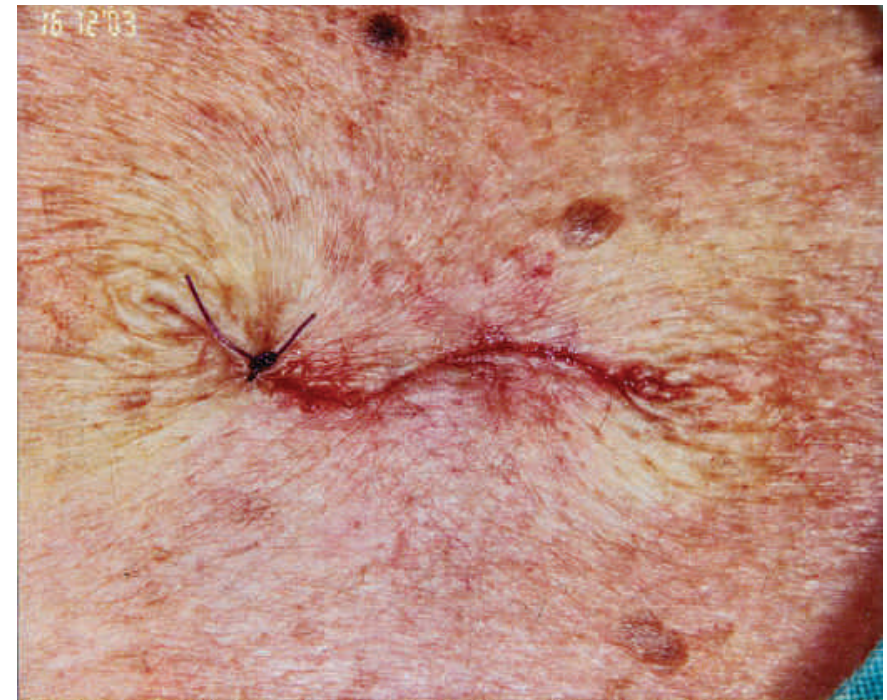

Abb. 4 Die S-förmige Nahtlinie

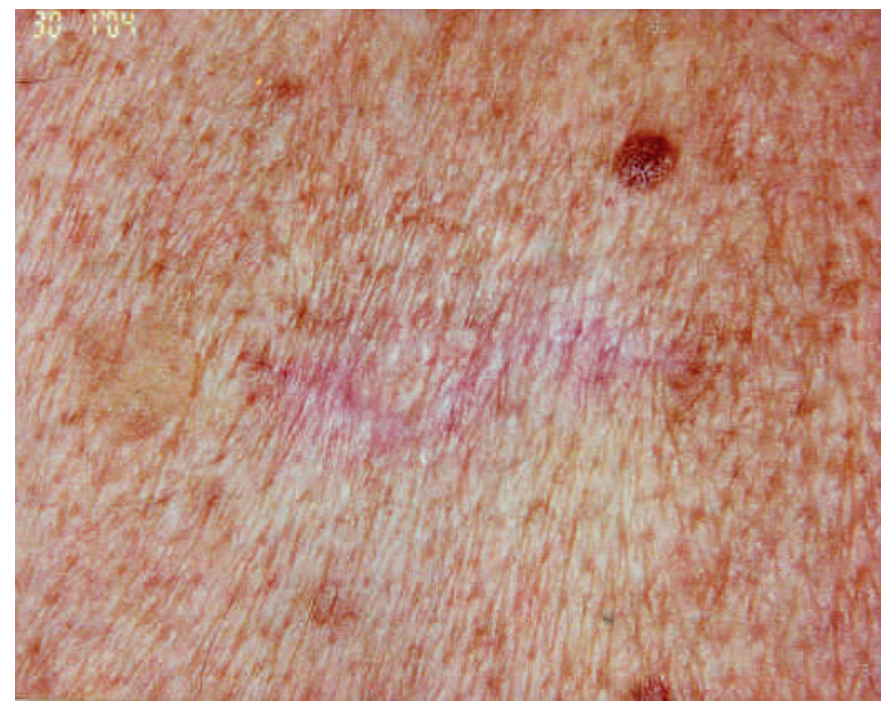

Abb. 5 Die S-förmige Narbe nach 7 Wochen

\section{Kasuistik}

Der Patient ist ein 59-jähriger Mann mit zwei Basalzellkarzinomen am Rücken. Die Defekte sind rund und verbunden (Abb. 2). Nach der Präparation der zwei Lappen werden sie in die Defekte verschoben (Abb.3) und mit subkutanen Nähten fixiert (resorbierbar, monofil, Polydioxanon) (Abb. 4). Der postoperative Heilungsverlauf war komplikationslos.

\section{Diskussion}

Die Verschiebelappenplastik nach von Burow ist eine häufig angewandte und vielfältig einsetzbare Technik in der operativen Dermatologie [1,2]. Boggio et al. [3] und Kaufmann und Landes $[4,5]$ haben die Verschiebelappenplastik nach von Burow für die Exzision von zwei nahen Hautläsionen beschrieben. 
Wir stellen hier einen Fall vor mit einer Modifikation dieser Technik bei runden und verbundenen Defekten (Abb. 3). Die Vorteile dieser Modifikation im Vergleich zur bisherigen Vorgehensweise (elliptische Exzision oder traditionelle Burow-Verschiebelappenplastik) liegen auf der Hand. Mit beiden bisherigen Techniken sind mehr Inzisionen notwendig und gesunde Haut muss exzidiert werden (d.h. Ausgleichsdreiecke).

Darüber hinaus sind die kürzere Operationszeit und die weniger invasive Vorgehensweise von Vorteil. Ein gutes ästhetisches Ergebnis lässt sich durch das Verstecken der S-förmigen Nahtlinie in den „relaxed skin tension lines“ erzielen (Abb.5).
Literatur

${ }^{1}$ Petres J, Rompel R. Operative Dermatologie. Berlin: Springer, 1996

2 Robinson JK, Arndt KA, Leboit PE, Wintroub BU. Atlas of cutanous surgery. Philadelphia, London, Toronto, Montreal, Sydney, Tokyo: W.B. Saunders Company, 1996

${ }^{3}$ Boggio P, Gattoni M, Zanetta R et al. Burow's triangle advancement flaps for excision of two closely approximated skin lesions. Dermatol Surg 1999; 25 (8): $622-625$

${ }^{4}$ Kaufmann R, Landes E. Simultaneous excion of two basal cell carcinomas. In: Kaufmann R (ed). Interventi dermochirurgici. Milano: Masson, 1994: 133

${ }^{5}$ Kaufmann R, Landes E. Simultaneous excion of two pigmented lesions. In: Kaufmann R (ed). Interventi dermochirurgici. Milano: Masson, 1994: 217

\section{Buchbesprechung}

\author{
Schützendes Sonnenlicht. Die heilsamen Kräfte der Sonne \\ M. F. Hollick, M. Jenkins \\ Stuttgart: Karl F. Haug, 2005. 144 S. Geb. 14,95€. \\ ISBN 3-8304-2208-3
}

Der international anerkannte Forscher Michael Holick aus Boston/USA hat zusammen mit dem Medizinjournalisten Mark Jenkins im Jahre 2004 ein handliches Büchlein für das amerikanische Publikum unter dem Titel „The UV Advantage“ verfasst. Dabei haben sich die beiden Autoren bewusst gegen eine einseitige „Sonnenverteufelung“ gewandt, wie sie von eifrigen Kollegen zur Hautkrebsprophylaxe propagiert wird, nach dem Motto „Keine Sonne, bedeckende Kleidung und Lichtschutz mit hohen Schutzfaktoren“. Vielmehr haben sie versucht, die experimentellen, klinischen und epidemiologischen Forschungen darzustellen, welche die biopositiven Effekte von Sonne, in kontrollierter Exposition, belegen und rechtfertigen. Vor allem die photochemische Vit.D3-Synthese in der Haut scheint neben der Prophylaxe von Vit.D-Mangelkrankheiten der Knochen auch einen nicht zu unterschätzenden Einfluss zur „Kanzeroprotektion“ zu haben bei Malignomen von Prostata, Darm, Ovar und Brust. Aber auch bei andern Malignomen, ja sogar bei Lymphomen der Haut, und bei entzündlichen Krankheiten ist eine biopositive Wirkung von moderater Sonnenexposition festzustellen. Auch saisonale Verstimmungen (SAD) werden mit Licht aufgehellt. So weit werden die seit 1989 in 2-jährigem Abstand abwechselnd in Basel und USA (Atlanta oder Boston) vorgetragenen und diskutierten wissen- schaftlichen Ergebnisse zusammengefasst und allgemein zugðnglich formuliert. Es handelt sich also um einen Auszug und eine Zusammenfassung all dessen, was in den jeweiligen Proceedings „Biologic Effects of Light“ ausführlich publiziert wurde, mit vielen „Dafür und Dagegen“. Ein Buch also für jeden interessierten und besorgten Menschen, und für Patienten, und eine Gegengewicht gegen die extreme Anti-Sonnen-Kampagne in den USA. Licht und Lichtquellen werden vorgestellt, Sonnenlicht und Hautkrebs sowie Sonnenlicht und Hautalterung werden erörtert und all die biopositiven Lichteffekte argumentiert. Dazu kommen Verhaltensmaßregeln zum ausgewogenen Sonnenverhalten und zur Mitberücksichtigung individueller, saisonaler und klimatischer Korrekturen mit Tafeln für die Regionen der USA. Der journalistische Impetus ist oft deutlich, die Vehemenz der Sprache auch, und so wird offenbar der amerikanische Leser bestens angesprochen.

Nun liegt seit April 2005 eine deutsche Übersetzung vor, die rasche und weite Verbreitung erfährt. Dabei ist der Titel „Schützendes Sonnenlicht“ eher noch etwas provozierender gewählt worden und die Eigenheiten amerikanischer Journalistik wurden beibehalten. In der deutschen Übersetzung wirken sie etwas ungewohnt, ja befremdlich. Ich bedaure dies.

Ein interessantes, ja nötiges Unterfangen mit Wirkung. Hoffentlich dient es nicht nur der Provokation, sondern, und vor allem, zur ausgewogenen Information der Menschen und zur Versachlichung der Debatte.

E. G. Jung, Heidelberg 